American Journal of Applied Sciences 8 (6): 603-609, 2011

ISSN 1546-9239

(C) 2011 Science Publications

\title{
Determination of Scattering Parameters of a New Copolymer by Using a Laser Scattering Method
}

\author{
Riyad Ghazy \\ Department of Physics, Laser Laboratory, Faculty of Sciences, \\ Tanta University, Tanta, Egypt
}

\begin{abstract}
Problem statement: In present work a poly (4 vinylphenol-co-2 hydroxethyl methacrylate) polymer, (PVPh-HEM), was selected, as a new copolymer, to study the phenomena of Optical Laser Light Scattering (OLLS). Approach: A poly (4 vinylphenol-co-2 hydroxethyl methacrylate) was investigated for its importance in the highly industrial applications in different branches. This polymer was solved in two different solvents like as Methanol and Dimethylformamide, DMF, with different concentrations $0.2,0.4,0.6,0.8,1.0 \times 10-3 \mathrm{~g} \mathrm{~mL}^{-1}$. OLLS data were obtained by using a self- built of laser scattering system. Results: The angular behavior of that selected polymer solution with different concentrations were studied by plotting the intensity of the scattered light against the scattering angle. The value of $k c / R(\theta)$ ratio was plotted as a function of $\theta$ through the value $\sin ^{2}(\theta / 2)+100 \mathrm{C}$, showing what was called Zimm plot. Conclusion/Recommendations: From that plot the different scattering parameters such as Pap's molecular weight $\mathrm{Mw}$, Second virial coefficient $\mathrm{A}^{2}$, Radius of gyration RG and molecular radius $\mathrm{D}$ of that polymer were determined in that two different selected solvents.
\end{abstract}

Key words: Scattering parameters, laser scattering, molecular weight, refractive indices, polymer solution, scattered light, dilute solution, rayleigh ratio, small macromolecules

\section{INTRODUCTION}

A poly (4-vinylphenol-co-2 hydroxethyl methacrylate) is a new copolymer its density is $1.2 \mathrm{~g}$ $\mathrm{mL}^{-1}$ at $250 \mathrm{C}$ and its transition temperature is $700 \mathrm{C}$. This copolymer is used in electronics, solder resists, etching resists, presensitized printing plates, coatings and adhesives. The chemical formula of poly (4vinylphenol-co-2 hydroxethyl methacrylate) is shown in Fig. 1.

A poly (4-vinylphenol-co-2 hydroxethyl methacrylate) (PVPh-HEM) is used to improve the thermal, mechanical and spectroscopic properties of the other polymer materials like as poly (Ethylene Oxide) (PEO) to meet some new practical applications (Rocco, et al., 2003).

When a monochromatic and coherent light is incident in dilute macromolecules solution and if the solvent molecules and the solute macromolecules have different refractive indices, the incident light will be scattered by each illuminated macromolecule to all directions (Vollmert, 1973).

\section{MATERIALS AND METHODS}

The basic pieces of information yielded by a light scattering measurement with a dilute polymer solution of small macromolecules are the weight-average molecular weight and the second virial coefficient. For macromolecules larger than one twentieth of the wavelength of the incident light, i.e., for large macromolecules, in addition to those two parameters the z-average radius of gyration is obtained. Therefore, the scattering of visible light (wavelengths $400-700 \mathrm{~nm}$ ) from dilute polymer solution is one of the fundamental experimental methods of the physical chemistry of polymers. In the mode of what called, the static or classical light scattering, we get one of the few absolute methods (i.e., not requiring calibration) for the determination of molecular weight of macromolecular substances. In the following, the basic application of the static light scattering is discussed:



Fig. 1: The chemical formula of Poly (4 VinylPhenolco-2 HydroxEthylMethacrylate), (PVPh-HEM), polymer 
The phenomenon of the light scattering from dilute polymer solution is based on rigorous theory, which is a combination of physical optics and thermodynamics (Kratochvil, 1987; Brown, 1996). The theory is rather complex, as testified by the fact that it was developed by no lesser personages than Einstein and Debye (19947). In the theory of light scattering the concept of small and large particles is important.

Small particles are meant particles or macromolecules, which are much smaller than the wavelength of incident light. Such particles behave as point source of scattered light. In experimental practice, particles smaller than about one twentieth of the wavelength, i.e., smaller than 20-30 nm, can be treated as small particles. With large particles, i.e., those larger than approximately one twentieth of the wavelength of light, the scattering phenomenon is affected by the intraparticle or intramolecular interference.

\section{Theory:}

Light scattering from small particles: Molecular parameters of a polymer can be obtained from its contribution to the overall intensity of light scattered from its dilute solution. This contribution equals the difference between the scattering intensities of the solution and pure solvent. In order to eliminate the influence of specific experimental conditions, such as intensity of incident beam, its polarization, geometry of the apparatus, a quantity for the normalized scattered light intensity, called the Rayleigh Ratio of symbol $\left(\mathrm{R}_{\theta}\right)$ has been defined. The subscript $(\theta)$ denoting the angle at which the scattering intensity has been measured.

At infinite dilution, the intensity of light scattered from a solution of uniform small macromolecules is described by the simple Equation (Huglen, 1972):

$\lim _{x \rightarrow \infty} \frac{k c}{R_{\theta}}=\frac{1}{M_{w}}$

where:

$\mathrm{c} \quad=$ Concentration of the solute

$\mathrm{M}_{\mathrm{w}}=$ Molecular weight

$K=\frac{2 \pi^{2} n_{0}^{2}}{\lambda^{4} N_{A}}\left(\frac{d n}{d c}\right)\left(1+\cos ^{2} \theta\right)$

Where:

$\mathrm{n}_{\mathrm{o}}=$ Refractive index of the solvent

$\lambda=$ Wavelength in vacuum of the incident light

$\mathrm{N}_{\mathrm{A}}=$ Avogadro's number

$\mathrm{dn} / \mathrm{dc}=$ The specific refractive index increment
The value of $\mathrm{k}$ depends on the polymer, solvent and temperature. The dependence of $\mathrm{k}$ on the high, fourth negative power of the wavelength indicates that the light with short wavelength, e.g. blue is scattered much more intensely than the light with long wavelength, e.g. red. For highly dilute solutions, the differential ratio can be replaced with no loss of accuracy by the ratio (.n/c), which is a quantity easy to measure, where .n is the difference between the refractive indices of the solution and solvent. As the right-hand side of Eq.1 does not contain any term depending on the scattering angle, the Rayleigh ratio for solutions of small macromolecules does not depend on the angle of observation, i.e., any angle can be used for the measurement.

With molecular solutions, the colloidal particles consist of small gel particles, i.e., the coiled chain molecules which have become solvated with solvent to extent that the entire gel particles consists of 90 to $99 \%$ of solvent. In this case, the refractive index of the solution differs slightly from the solvent refractive index. This gives rise to simple physical laws for the scattering of light (Siddiq et al., 1997; 1998).

The basic equation describing the light scattering from dilute solutions of nonuniform small macromolecules according to $\mathrm{P}$. Debye is usually written as:

$\frac{\mathrm{kc}}{\mathrm{R}_{\theta}}=\frac{1}{\mathrm{M}_{\mathrm{w}}}+2 \mathrm{~A}_{2} \mathrm{c}+\left(3 \mathrm{~A}_{3} \mathrm{c}^{3}+\ldots \ldots\right)$

And:

$\mathrm{R}_{\theta}=\frac{\mathrm{I}_{\theta} \mathrm{r}^{2}}{\mathrm{I}_{0} \mathrm{~V}}$

With Mw the weight average molecular weight:

$\mathrm{V}=$ Scattering volume

$\mathrm{A}_{2}=$ Second virial coefficient (Paul et al., 19987; Remsen, 1991)

$\mathrm{R}=$ Distance between the observer and the scattering volume

$\mathrm{I}_{\mathrm{o}}=$ Incident intensity

$\mathrm{I}_{\theta}=$ Scattered intensity

In Eq. $3 \mathrm{~A}_{2}$ and $\mathrm{A}_{3}$ are the second and third virial coefficients, respectively. The second virial coefficient is an important thermodynamic characteristic of the polymer-solvent system. High values of $\mathrm{A}_{2}$ are characteristics of systems with intense interaction 
between the polymer and solvent, that means of a good solvent for the given polymer. The term with virial coefficients in Eq. 3 describes the effect of the interference of light scattered by different macromolecules, i.e., intermolecular interference, on the intensity of light scattered from the solution.

To determine the molecular weight of a polymer which molecules are small $\lambda / 20$ (where $\lambda$ is the wavelength), we measure the intensity of light scattered from two to four solutions with different concentrations, calculate the ratio $\mathrm{kc} / \mathrm{R}_{\theta}$, most frequently $\mathrm{kc} / \mathrm{R}_{90}$ and plot them as a function of polymer concentration. The intercept with the axis of ordinate gives the reciprocal value of $\mathrm{Mw}$ according to Eq. 1 (Shere et al., 1996) and the slope of the concentration dependence being twice the value of $A_{2}$.

In this simple way, i.e., through the measurement of scattered light intensity at a single angle $\theta$, the molecular weight can be obtained but only for particle diameters smaller than $\lambda / 20$, which is only found in a number of proteins and linear polymers. For such polymer, any observation angle can be selected since the scattering function is symmetrical. However, for experimental reasons, it is usually selected at $90^{\circ}$ (Patterson and Jamieson, 1985; Wu et al., 1996).

Light scattering from large particles: Light scattering from various mass points of a large particle reaches the detector with different phase and interferes giving rise to the phenomenon of intraparticle or intramolecular interference, which results in a decrease in the intensity of scattered light. There is just one direction at which no interference occurs no matter what the size of the particle is, this is the direction of the incident beam, i.e., at angle of observation zero.

Unfortunately, at this angle no measurement can be made, because the weak scattered beam is virtually lost in the primary beam, of intensity larger by many orders of magnitude. For practically all polymer molecules occurring in common experimental practice, the extent of interference increases with increasing angle of observation. Unlike for small particles, for large particles the intensity of scattered light depends on the angle of observation. The angular dependence of the scattered intensity is pre-determined by the size and shape of the scattering particles. Thus, at least of principle, it must be possible to obtain some information on the size and shape of the scattering particles from the angular dependence of scattering intensity (Ghazy et al., 1999 Moreels et al., 1997; Bender and Lewis, 1986' Chau and Rudin, 1973). The factor by which the scattering intensity $I_{\theta}$ is diminished by interference at an angle $\theta$ is called the scattering function $\mathrm{P}(\theta)$, which incorporates the effect of chain size and conformation on the angular dependence of scattered light intensity. In the case of spherical particles smaller than the wavelength act as independent scattering centers generating a symmetrical envelope of scattering light intensity. In this case of small particles, $\mathrm{P}(\theta)$ is unity. But in the case of polymer chains whose dimensions are $>\lambda / 20$, scattering may occur from different points along the same chain. While, the form $\mathrm{P}^{-1}(\theta)$ is dependent upon the size and the shape of the scattering particle and consequently provides the means for their determination (Stacey, 1956):

$$
\mathrm{P}^{-1}(\theta)=1+\frac{16 \pi^{2}}{3 \lambda^{2}}\left\langle\mathrm{R}_{\mathrm{G}}^{2}\right\rangle_{\mathrm{z}} \operatorname{Sin}^{2}\left(\frac{\theta}{2}\right)
$$

In which:

$\lambda=\lambda_{\mathrm{o}} / \mathrm{n}_{\mathrm{o}}=$ Wavelength of light in the medium

$\langle\mathrm{RG}\rangle=$ MEAN square radius of gyration, which is independent of the particle shape at small angles

The particle scattering function $\mathrm{P}(\theta)$ of randomlycoiling molecules, as they are usually found in a macromolecular solution, is dependent on the scattering angle $\theta$ as on the wavelength and on a quantity called the radius of gyration.

The radius of gyration $R_{G}$, is defined by the relation:

$\mathrm{R}_{\mathrm{G}}=\left[\frac{\sum \mathrm{m}_{\mathrm{i}} \mathrm{r}_{\mathrm{i}}}{\sum \mathrm{m}_{\mathrm{i}}}\right]^{1 / 2}$

Where, $m_{i}$ and $r_{i}$ are the weight of mass point $i$ of the particle and its distance from the center of the particle, respectively.

Thus, the radius of gyration is the square root of the mean square distance of the mass unit from the center of mass of the particle. Then, it is a general size parameter, because its definition does not depend on the shape of the particle. Unlike size parameters specific for individual particle shapes, such as radius or diameter of a sphere, length of a thin rod, or end-to-end distance of a random coil. On the other hand, these specific size characteristics are much more graphic than the radius of gyration. The two types of quantities are easily interconvertible using simple probability relations.

The basic equation describing light scattering from dilute solutions of non-uniform large macromolecules at finite concentrations is usually written in the form:

$$
\begin{aligned}
& \frac{\mathrm{kC}}{\mathrm{R}_{\theta}}=\frac{1}{\mathrm{M}_{\mathrm{w}}}\left[1+\frac{16 \pi^{2}}{3 \lambda^{2}}\left\langle\mathrm{R}_{\mathrm{G}}^{2}\right\rangle_{\mathrm{z}} \sin ^{2}\left(\frac{\theta}{2}\right)+\ldots . .\right] \\
& +2 \mathrm{~A}_{2} \mathrm{C}+3 \mathrm{~A}_{3} \mathrm{C}^{3}+\ldots . .
\end{aligned}
$$


Where, $\left\langle R G^{2}\right\rangle_{\mathrm{Z}}$ is the square of the $\mathrm{z}$-average radius of gyration and the angle brackets indicate that for flexible macromolecules the value has to be averaged over all possible conformation and the other symbols have the meanings defined above.

For large angles, the form of $\mathrm{P}(\theta)$ varies according to the shape of the scattering particles and has been evaluated for common shapes, like, uniform shapes (Rayleigh, 1899; 1903) thin rods (Nengebauer, 1942), Gaussian coils which are monodispers (Debye, 1947) and polydisperse (Zimm, 1948).

Details of such particle scattering function are given Huglen, (1972) but it is interested to restate the derived results in terms of the characteristic dimension $\mathrm{D}$ of the scattering particle:

For Rods $D^{2}=12\left\langle R^{2} G\right\rangle$

For Spheres $D^{2}=5\left\langle R^{2} G\right\rangle$

For flexible coil $D^{2}=6\left\langle R^{2} G\right\rangle$

In order quoted, D represents the length of the rod, the radius of the sphere and the root mean square endto-end distance of the coil. In the case of polymer molecules are treated as a flexible coil, then Eq. 7 can be written in the form:

$$
\frac{\mathrm{kC}}{\mathrm{R}(\theta)}=\frac{1}{\mathrm{M}_{\mathrm{w}}}\left[1+\frac{8 \pi^{2}}{9 \lambda^{2}} \mathrm{D}^{2} \sin ^{2}\left(\frac{\theta}{2}\right)\right]+2 \mathrm{~A}_{2} \mathrm{C}+3 \mathrm{~A}_{3} \mathrm{C}^{3}+
$$

The first term on the right-hand side of Eq. 7 describes the angular dependence of the ratio $(\mathrm{kc} / \mathrm{R})$ at infinite dilution. The second and potential further terms describe the concentration dependence of $(\mathrm{kc} / \mathrm{R})$ at zero angle of observation because for $\theta=0$ the series in parentheses reduces to unity and Eq.7 assumes the form of Eq. 3. The second term in parentheses depends on the $\mathrm{z}$-average radius of gyration of the scattering macromolecules, which means dependence on their size but not on their shape. Only the third and further molecules are too small to enable us to reliably determine the shape of the scattering molecules.

Equation 7 tells that, in order to determine the molecular weight, the z-average radius of gyration and the second virial coefficient of a polymer solute in a given solvent, angular dependences of the scattering intensity have to be measured for several (usually three to four) solutions with different concentrations. Then, the values of the ratio $(\mathrm{kc} / \mathrm{R})$ are calculated for all experimental points and two sets of dependences are constructed, the angular dependences of $(\mathrm{kc} / \mathrm{R})$ at constant concentrations and the concentration dependences of $(\mathrm{kc} / \mathrm{R})$ at the individual angles of measurement.
The angular dependences are extrapolated to zero angle of measurement to eliminate the intramolecular interference and the concentration dependences are extrapolated to infinite dilution, i.e., to zero concentration, to eliminate the intramolecular interference. Through the extrapolated points, two extrapolated dependences are plotted, viz., the extrapolated angular dependence for infinite dilution and the extrapolated concentration dependence for zero angle of measurement. Both extrapolations are conveniently made graphically in one diagram called the Zimm plot. The two extrapolations provide the most important information that we need.

The ordinate of the common intercept of the two extrapolations with the $y$-axis is the reciprocal value of the average molecular weight of the polymer we investigate. The initial slope of the extrapolated angular dependence for infinite dilution is proportional to the $\mathrm{z}$ average radius of gyration and the initial slope of the concentration dependence for zero angle of measurement is proportional to the second virial coefficient (Frato, 2003).

In general, it can be taken as a rule: For polymers with small chain macromolecules with molar masses smaller than $\left(2 \times 10^{5}\right)$, a standard light scattering measurement yields the average molecular weight and the second virial coefficient.

Chain macromolecules in the molecular weight between, approximately, $\left(2 \times 10^{5}\right)$ and $\left(1 \times 10^{7}\right)$, behave as large particles for which the molecular weight, the $\mathrm{z}-$ average radius of gyration and the second virial coefficient can be determined.

For our laser light scattering measurements, the chain molecules of the polymers that we used are of diameters larger than $(\lambda / 20)$. Therefore, the molecular weight must be determined by measuring the dependence of scattered light intensity on the scattering angle.

Zimm plot: The Zimm treatment (Zimm, 1948) affords the most accurate graphical procedure for the derivation of light scattering parameters when angular dependence comes into play.

It is seen from Eq. 7 that the factor $(\mathrm{kc} / \mathrm{R})$ has a linear dependence on the concentration $\mathrm{c}$ (for constant angle of observation) and a linear dependence on $\sin ^{2}$ $(\theta / 2)$ \{if the concentration $\mathrm{c}$ is held constant $\}$.

The first step in the construction of Zimm plot is to carry out angular distribution of light scattering measurement at different constant concentrations from $30-150^{\circ}$ as shown in Fig. 4. The results obtained are plotted graphically as $(\mathrm{kc} / \mathrm{R})$ versus $\mathrm{c}$ at constant angle $\theta$ and $(\mathrm{kc} / \mathrm{R})$ versus $\sin 2(\theta / 2)$ at constant concentration c.

That graphical representation yields a series of straight lines which can be extrapolated to the ordinate and therefore, permit determination of $(\mathrm{kc} / \mathrm{R})$ at $\mathrm{c} \rightarrow 0$ and $(\mathrm{kc} / \mathrm{R})$ at $\theta \rightarrow 0$. 
Therefore, in Eq. 7.

At $\mathrm{c} \rightarrow 0$, we can get the form:

$$
\frac{\mathrm{kC}}{\mathrm{R}_{\theta}}=\frac{1}{\mathrm{M}_{\mathrm{w}}}\left[\frac{1}{\mathrm{M}_{\mathrm{w}}}+\frac{16 \pi^{2}}{3 \lambda^{2}}\left\langle\mathrm{R}_{\mathrm{G}}^{2}\right\rangle \sin ^{2}\left(\frac{\theta}{2}\right)\right]
$$

And also at $\theta \rightarrow 0$, the equation will be in the form:

$\frac{\mathrm{kC}}{\mathrm{R}_{\theta}}=\frac{1}{\mathrm{M}_{\mathrm{w}}}+2 \mathrm{~A}_{2} \mathrm{c}$

According to the above equations, the values of $(\mathrm{kc} / \mathrm{R})$ as a function of the concentration $\mathrm{c}$ at constant angle of observation $\theta$ and $(\mathrm{kc} / \mathrm{R})$ as a function of the angle $\theta$ at constant concentration $\mathrm{c}$ are obtained. Then, they are represented in a new graph (Zimm graph) as straight lines.

In the same single diagram, one plot $(\mathrm{kc} / \mathrm{R})$ versus $\sin ^{2}(\theta / 2)+K c$. Choosing the value $K$ to make the concentration term comparable with $\sin ^{2}(\theta / 2)$ and extrapolate the resulting lines at constant concentration and constant angle to give a grid. The extrapolated points at different angles for zero concentrations (according to Eq. 9) are extrapolated to zero angle, i.e., to cut the $\mathrm{kc} / \mathrm{R}$ axis and similarly the points of zero angle at different concentration (according to Eq. 10) are extrapolated to zero concentration. Ideally, the two extrapolations should cut the $(\mathrm{kc} / \mathrm{R})$ axis at the same point. The reciprocal of the intercept, $[\mathrm{kc} / \mathrm{R}][\mathrm{kc} / \mathrm{R}]_{\mathrm{c}=0}^{\theta=0}$ is equal to the weight average molecular weight of the polymer.

The initial gradient of the graph at zero angles gives a measure of the second virial coefficient $\mathrm{A}_{2}$. Thus, $A_{2}=1 / 2$ (Initial slope of $\theta=0$ line). The mean square radius of gyration $\left\langle\mathrm{R}_{\mathrm{G}}^{2}\right\rangle$ is given by the intercept and initial slope of the line $\mathrm{c}=0$ (Li et al., 1997; Wu, 1997).

Experiment: The details of the experimental arrangement concerning to the phenomena of the static laser light scattering was explained in the literature (Ghazy et al., 1999; 2009).

\section{RESULTS AND DISCUSSION}

Figures 2-3 represent the angular distribution of the scattering intensities with two different solvents, the methanol and DMF.

For both solvents, a plot of $(\mathrm{kc} / \mathrm{R})$ as a function of the concentration is constructed at constant observation angle; corresponding to those $(\theta)$ values we obtain straight lines. Also, a plot of $(\mathrm{kc} / \mathrm{R})$ at constant concentration as a function of $\sin ^{2}(\theta / 2)$ is constructed and also yield straight lines. In both plots the straight lines are extrapolated to the $(\mathrm{kc} / \mathrm{R})$ axis to get the values of zero lines. As shown in Figs. 4-5 for Methanol and Figs. 6-7 for DMF.

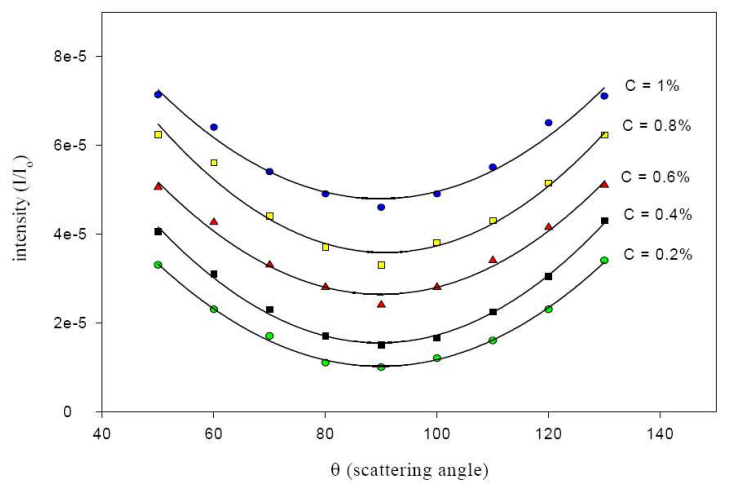

Fig. 2: The angular distribution of light scattering measurements for Poly (4-vinylphenol.co-2 hydroxethyl methacrylate) Solved in Methanol at different concentrations

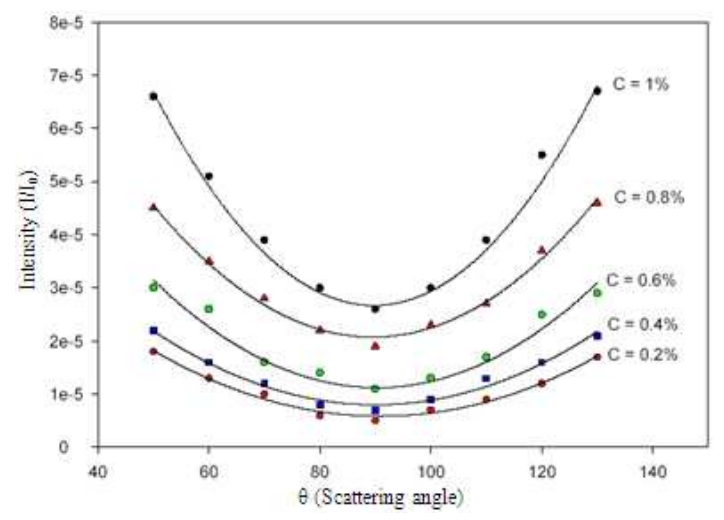

Fig. 3: The angular distribution of light scattering measurements for Poly (4-vinylphenol-co-2 hydroxethyl methacrylate) Solved in DMF at different concentrations

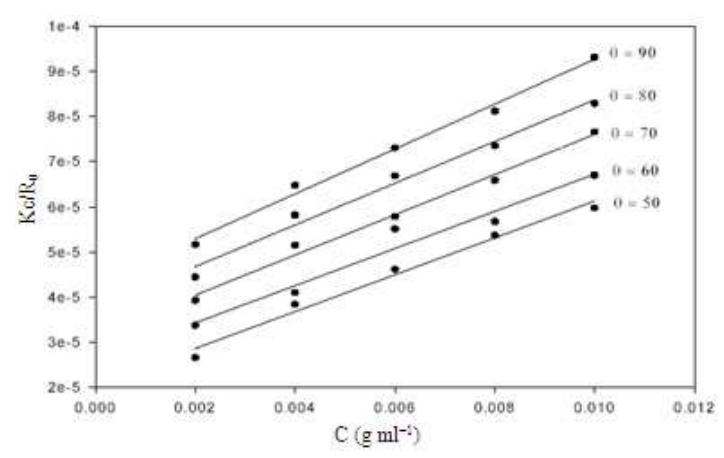

Fig. 4: kc/R $\theta$ of poly (4-vinylphenol-co-2 hydroxethyl methacrylate), solved in methanol, as a function of the concentration $\mathrm{c}$ at constant angle 


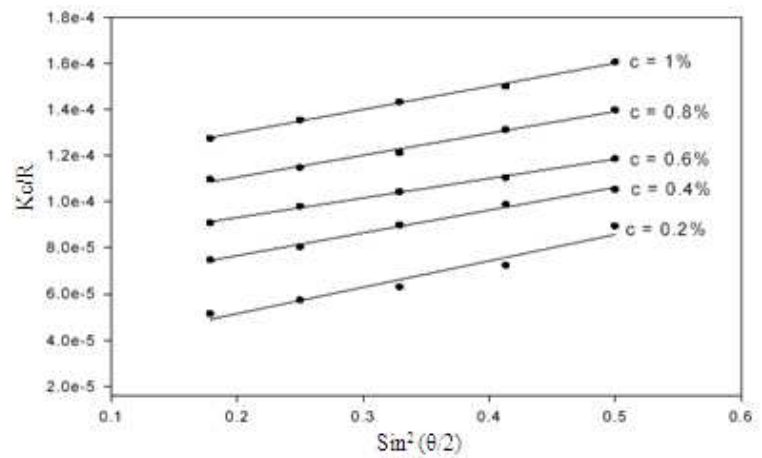

Fig. 5: kc/R $\theta$ of poly(4-vinylphenol-co-2 hydroxethyl methacrylate) solved in methanol, as a function of the observation angle at constant concentration

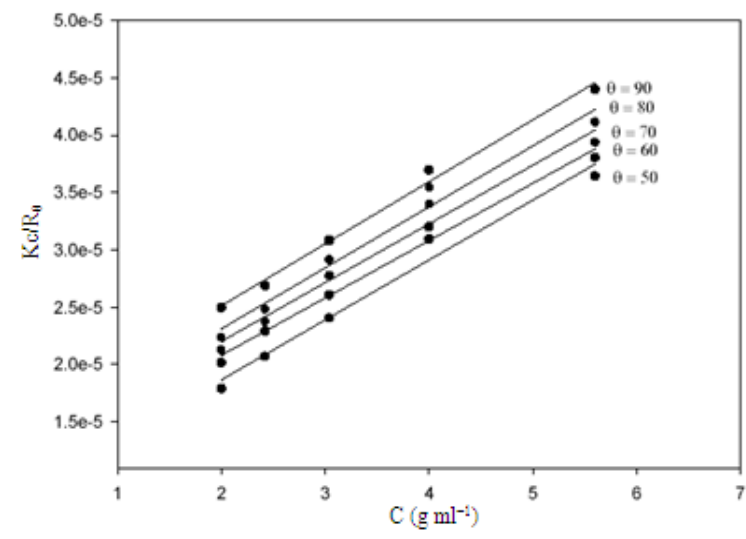

Fig. 6: kc/R $\theta$ of poly(4-vinylphenol-co-2 hydroxethyl methacrylate), solved in DMF, as a function of the concentration $\mathrm{c}$ at constant angle

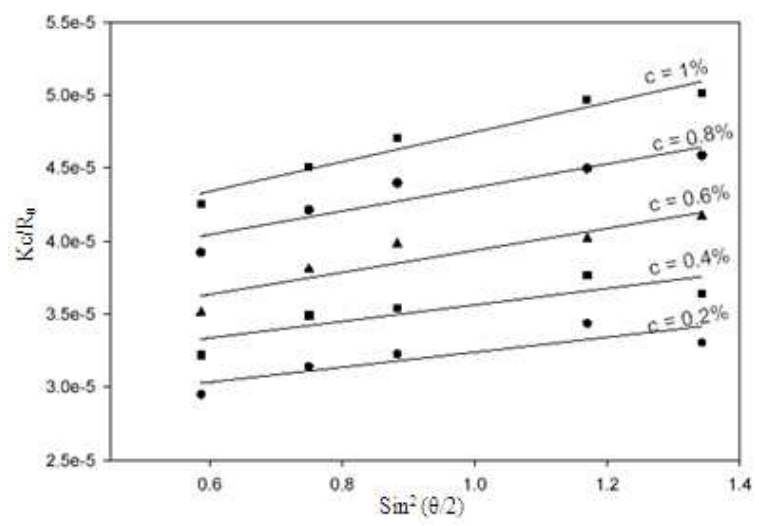

Fig. 7: kc/R $\theta$ of poly(4-vinylphenol-co-2 hydroxethyl methacrylate), solved in DMF, as a function of the observation angle at constant concentration $\mathrm{c}$

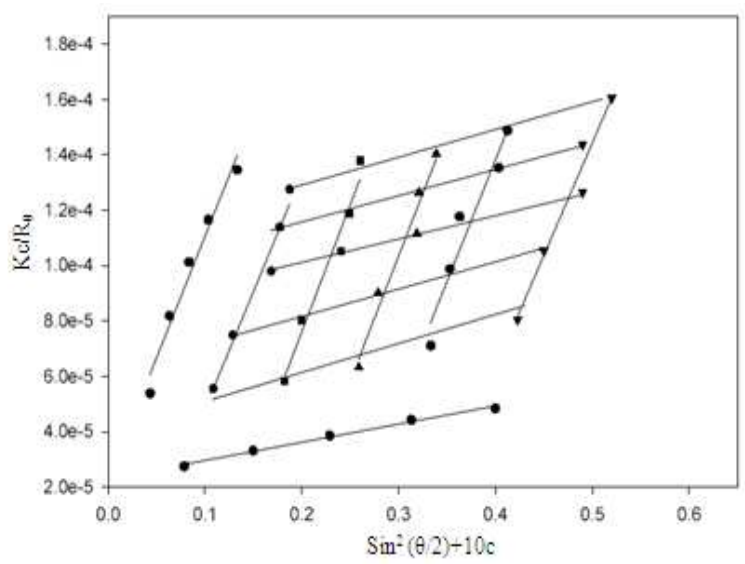

Fig. 8: Zimm plot of poly (4-vinylphenol-co-2 hydroxethyl methacrylate) solved in methanol

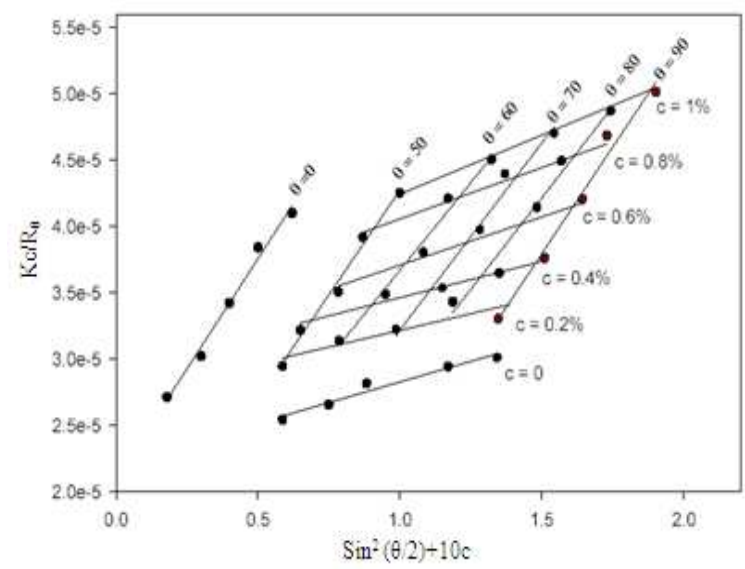

Fig. 9: Zimm plot of poly (4-vinylphenol-co-2 hydroxethyl methacrylate) solvedin DMF

Zimm plot can be drawn for the both solvents by using 5 different concentrations of solutions and 5 scattering angles. This makes a grid of 25 data The extrapolation values for $(\mathrm{kc} / \mathrm{R}) \mathrm{c}=0$ versus the $\sin ^{2}(\theta / 2)$ (at $\mathrm{c}=0$ ) values are plotted. Also the extrapolated values from $(\mathrm{kc} / \mathrm{R}) \theta=0$ are plotted versus the concentration $c$, at $\sin ^{2}(\theta / 2)=0$. To obtain two straight lines which represent the zero lines. The intercept of this two lines with the ordinate yield $(1 / \mathrm{Mw})$. From the slope of line at $\mathrm{c}=0$, we can obtain the value of $\mathrm{RG}$ and the initial slope of line $\sin ^{2}(\theta / 2)=0$ is equal to $2 \mathrm{~A} 2$. Zimm plots are shown in Fig. 8 for Methanol and Fig. 9 for DMF, respectively. Table 1 shows the obtained values. 
Am. J. Applied Sci., 8 (6): 603-609, 2011

\begin{tabular}{|c|c|c|c|c|}
\hline \multirow[b]{2}{*}{ Solvents } & \multicolumn{4}{|c|}{ Measured values at $\lambda=488 \mathrm{~nm}$} \\
\hline & $\mathrm{M}_{\mathrm{w}}\left(\mathrm{g} \mathrm{mol}^{-1}\right)$ & $\mathrm{A}_{2}\left(\right.$ mol.mL g $\left.{ }^{-2}\right)$ & $\mathrm{R}_{\mathrm{G}}(\mathrm{nm})$ & $\mathrm{D}(\mathrm{nm})$ \\
\hline Methamol & 44748.18 & $4.39640 \mathrm{e}-4$ & 115 & 282 \\
\hline DMF & 45284.52 & $1.65936 \mathrm{e}-4$ & 113 & 277 \\
\hline
\end{tabular}

\section{CONCLUSION}

We have constructed an experiment for measuring the angular distribution of light scattered intensity at scattering angles from $\left(40-150^{\circ}\right)$. The angular distributions of scattered intensity of poly (4 vinylphenol-co-2 hydroxethyl methacrylate) in two different solvents; Methanol and DMF at five different concentrations. By using this data in a Zimm plot we calculated the molecular weight of polymer at the two solvents. Also another important information is calculated using the same Zimm plot that is the second virial coefficient $A_{2}$ for the two solvents. The large value of A2 for Methanol indicates that it is the better as a solvent for this polymer. The radius of gyration at the two solvents is also calculated. This proves that one can use this experiment to determine the precise value of the molecular weight for unknown polymers.

\section{REFERENCES}

Bender, T.M. and R.J. Lewis, 1986. Macromolecules, 19: 244.

Brown, W., 1996. Light Scattering Principle and Development. Clarendon Press, Oxford.

Chau, T.C. and A. Rudin, 1973. J. Polymer, 15: 593.

Debye, P.J., 1947. Phys. Colloid Chem., 51: 18, 1947.

Frato, K.E., 2003. Physics Department. College of Wooster, Ohio 44691.

Ghazy, R., B.El-Baradie, A.El-Shaer and F.El-Mekawy, 1999. Optics Laser Technol., 31: 447-453.

Ghazy, R., N. Fotoh, B. Moharam and F. El-Mekawey, 2009. On physics of Optical Laser Light Scattering (OLLS) of an industrial polymer which may assist in physics of Random Laser (RL) investigation. J. Light Vis. Environ., 33: 13-23. http://www.sigmaaldrich.com/materialsscience/material-scienceproducts.html? TablePage $=9539178$
Huglen, M.B., 1972. Light Scattering from Polymer Solutions. Academic PressINC(London) Ltd.

Huglen, M.B., 1972. Light Scattering from Polymer Solutions. Academic Press INC (London) Ltd.

Kratochvil, P., 1987. Classical Light Scattering from Polymer Solutions. Elsevier,Amsterdam, 1987.

Li, M., M. Jing, L. Zhu and C.Wu, 1997. Macromolecules, 30: 2201.

Moreels, E., W. De Cenuiuck and R. Finsy, 1987. J.Chem.Phys., 86: 618.

Nengebauer, T., 1942. Ann. Phys. Lepsig, 42: 509.

Patterson, P.M. and A.M. Jamieson, 1985. Macromolecules, 18: 266.

Paul, C.W. and P.M. Cotts, 1987. Macromolecules, 20: 1986.

Rayleigh, L., 1899. Scientific Papers.vol. I, University Press, Cambridge, pp: 518-536.

Rayleigh, L., 1903. Scientific Papers. vol. IV, University Press, Cambridge, pp: 397-405.

Remsen, E.E., 1991. J.Applied Polymer Sci., 42: 503.

Rocco, A., C. Bielschowsky and R. Pereira, 2003. Polymer. 44: 361.

Shere, A.J., M. Sethumadhavan, R. Stein, R.F. Saraf and R.A.Gaudiana et al., 1996. Macromolecules, 29: 2088 .

Siddiq, M., B. Li and C. Wu, 1997. J. Polymer Sci., 35: 85 .

Siddiq, M., B.Li and C. Wu, 1998. J. Polymer Sci., 63: 1755.

Stacey, K.A., 1956. Light Scattering in Physical Chemistry. LondonButter worth scientific publications.

Vollmert, B., 1973. Polymer Chemistry, Translated from the german by Edund H.Immergut. SpringerVerlag, New York,Berlin.

Wu, C., 1997. Polymer Adv. Technol., 8: 177.

Wu, C., K. Woo and M. Jiang, 1996. Macromolecules, 29: 536.

Zimm, B.H., 1948. J.Chem.Phys., 16: 1099-1116. 